\title{
Universal Lower Bound for Finite-Sample Reconstruction Error and Its Relation to Prolate Spheroidal Functions
}

\author{
Talha Cihad Gulcu ${ }^{\circledR}$, Member, IEEE, and Haldun M. Ozaktas, Fellow, IEEE
}

\begin{abstract}
We consider the problem of representing a finiteenergy signal with a finite number of samples. When the signal is interpolated via sinc function from the samples, there will be a certain reconstruction error since only a finite number of samples are used. Without making any additional assumptions, we derive a lower bound for this error. This error bound depends on the number of samples but nothing else, and is thus represented as a universal curve of error versus number of samples. Furthermore, the existence of a function that achieves the bound shows that this is the tightest such bound possible.
\end{abstract}

Index Terms-Finite-energy signals, nonbandlimited signals, prolate spheroidal functions, reconstruction error, sampling theory, unbandlimited signals, uncertainty principle, uncertainty relationship.

\section{INTRODUCTION}

W HEN we represent a signal with a finite number of samples, as is always the case in practice, there will be an inevitable reconstruction error when we attempt to recover the original signal by interpolating the samples. In this letter, we derive the minimum possible value for this error as a function of the number of samples. The error does not depend on any other problem parameters. The only assumption made is that the original signal has finite energy, which is always true for real physical signals. We do not assume the signal to have finite extent or to be bandlimited. Thus, we present a very simple, mostly analytical solution to a very basic problem. A small sample of works that deals with sampling of nonbandlimited signals, and containing further references, includes [1]-[12]. A related concept is the number of degrees of freedom (DOF), which is most frequently employed in conjunction with wave propagation in optics, wireless, etc. [13]-[18]. The DOF is sometimes, but not always, the same as the space-bandwidth (or time-bandwidth) product [19], [20].

Manuscript received October 25, 2017; accepted October 31, 2017. Date of publication November 3, 2017; date of current version November 15, 2017. The associate editor coordinating the review of this manuscript and approving it for publication was Prof. Waheed U. Bajwa. (Corresponding author: Talha Cihad Gulcu.)

T. C. Gulcu was with the Department of Electrical and Computer Engineering, University of Maryland, College Park, MD 20742 USA. He is now with TUBITAK, Advanced Technologies Research Institute, Ankara TR-06800, Turkey (e-mail: tcgulcu@gmail.com).

H. M. Ozaktas is with the Department of Electrical Engineering, Bilkent University, Ankara TR-06800, Turkey (e-mail: haldun@ee.bilkent.edu.tr).

Color versions of one or more of the figures in this letter are available online at http://ieeexplore.ieee.org.

Digital Object Identifier 10.1109/LSP.2017.2769695
In Section II, we formulate the problem. After this, we review prolate spheroidal functions in Section III, which are then employed to prove the main result in Section IV.

\section{PROBLEM Formulation}

In this section, we will see that the error when we reconstruct a signal from a finite number of samples is approximately equal to the energy of the signal in the neglected tails of the function, and therefore that a bound on the energy in the tails gives us a bound on the reconstruction error.

Let $f: \mathbb{R} \rightarrow \mathbb{C}$ be a finite-energy field having continuous realizations. We also assume that the Fourier transform $F(\mu)=$ $\int_{-\infty}^{\infty} f(u) e^{-j 2 \pi \mu u} d u$ exists for almost all realizations of $f(u)$. We refer to $u$ as the space domain, but it could also be referred to as the time domain.

Now, say we have one realization of $f(u)$. We wish to represent it by a finite number of samples such that if it is then interpolated from these samples, the mean-square error between $f(u)$ and its reconstructed version will be as small as possible. $f(u)$ is not necessarily bandlimited, but to apply the sampling theorem we will confine it to some frequency interval $[-\Delta \mu / 2, \Delta \mu / 2]$, which amounts to neglecting the frequency content outside this interval and implies a sampling interval of $1 / \Delta \mu$. Likewise, in the space domain, since we want a finite representation, we will restrict our attention to samples of $f(u)$ that fall within the interval $[-\Delta u / 2, \Delta u / 2]$, which implies a total number of $\Delta u /(1 / \Delta \mu)=\Delta u \Delta \mu$ distinct sample values, a quantity known as the space-bandwidth product. Assuming $\Delta u$ and $\Delta \mu$ are sufficiently large and applying standard sinc interpolation to these samples, we obtain the reconstructed signal $f_{\Delta \mu}, \Delta u$ as [12]

$$
f_{\Delta \mu, \Delta u}(u)=\sum_{n=-\lfloor\Delta u \Delta \mu / 2\rfloor}^{\lfloor\Delta u \Delta \mu / 2\rfloor} f_{\Delta \mu}\left(\frac{n}{\Delta \mu}\right) \operatorname{sinc}(\Delta \mu u-n)
$$

where $f_{\Delta \mu}(u)$ is $f(u)$ bandlimited to the frequency interval $[-\Delta \mu / 2, \Delta \mu / 2]$. The reconstruction error becomes

$$
\begin{aligned}
\int_{-\infty}^{\infty}\left|f(u)-f_{\Delta \mu, \Delta u}(u)\right|^{2} d u \approx & \int_{|u|>\Delta u / 2}|f(u)|^{2} d u \\
& +\int_{|\mu|>\Delta \mu / 2}|F(\mu)|^{2} d \mu
\end{aligned}
$$


where the approximation sign has the meaning that the ratio of left- and right-hand sides approaches to 1 as $\Delta \mu \rightarrow \infty$ [12].

The notation of $\sim$ under $\Delta \mu$ indicates confinement to that extent is in the opposite domain to which the signal is currently being represented in and is maintained for consistency with [12]. A dual result is also possible by first confining $f(u)$ to $[-\Delta u / 2, \Delta u / 2]$ in space and then sampling the Fourier transform of the resultant space-limited signal in the frequency interval $[-\Delta \mu / 2, \Delta \mu / 2]$. In this case, we similarly obtain [12]

$$
\begin{aligned}
\int_{-\infty}^{\infty}\left|f(u)-f_{\Delta u, \Delta \mu}(u)\right|^{2} d u \approx & \int_{|u|>\Delta u / 2}|f(u)|^{2} d u \\
& +\int_{|\mu|>\Delta \mu / 2}|F(\mu)|^{2} d \mu .
\end{aligned}
$$

So, regardless of whether we sample the signal in the space or frequency domain, in both cases the reconstruction error is approximately equal to the truncation error (the sum of the energies "left out" in the tails of the signal in the space and frequency domains), a result which also has numerical support [21], [22]. What we choose to neglect in the tails as a result of our choice of $\Delta u$ and $\Delta \mu$ returns to us as a reconstruction error.

The purpose of this letter is to investigate how small it is possible to make the common right-hand sides (RHS) of (2) and (3). Note that a converse problem is considered in [1, Th. 2], which provides a lower bound of $\Delta u \Delta \mu$ for a given $\int_{|u|>\Delta u / 2}|f(u)|^{2} d u$ and $\int_{|\mu|>\Delta \mu / 2}|F(\mu)|^{2} d \mu$. More precisely, we will prove that there is a value $\kappa(\Delta u \Delta \mu)$, depending only on the product $\Delta u \Delta \mu$ such that

$$
\begin{gathered}
\int_{|u|>\Delta u / 2}|f(u)|^{2} d u+\int_{|\mu|>\Delta \mu / 2}|F(\mu)|^{2} d \mu \\
\geq \kappa(\Delta u \Delta \mu) \int_{-\infty}^{\infty}|f(u)|^{2} d u
\end{gathered}
$$

with equality for a single function $f(u)$. We observe that the lower bound on the normalized reconstruction error depends only on the space-bandwidth product $\Delta u \Delta \mu$ and obtain an expression for the function $\kappa(\Delta u \Delta \mu)$.

It remains to prove (4). But first we review prolate spheroidal functions [23]-[30].

\section{PROlate Spheroidal FunCtions}

We first define the projection operators [26] needed to describe prolate spheroidal functions.

Definition 1: The projection operator $A$ confines the signal $f$ to the interval $[-\Delta u / 2, \Delta u / 2]$ :

$$
A f(u)=\left\{\begin{array}{cl}
f(u), & \text { if }|u| \leq \Delta u / 2 \\
0, & \text { else. }
\end{array}\right.
$$

Definition 2: The projection operator $B$ confines the Fourier transform $F$ of the signal $f$ to the interval $[-\Delta \mu / 2, \Delta \mu / 2]$ :

$$
B f(u)=\int_{-\Delta \mu / 2}^{\Delta \mu / 2} F(\mu) e^{j 2 \pi \mu u} d \mu .
$$

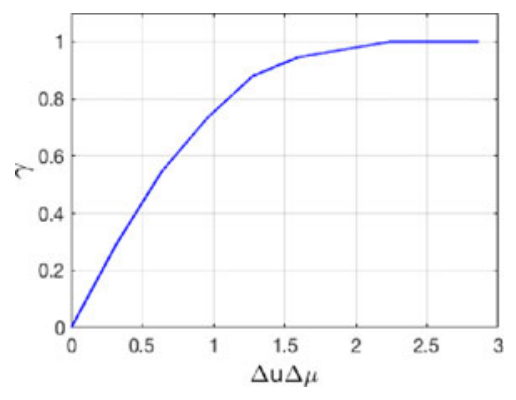

Fig. 1. $\gamma$ versus $\Delta u \Delta \mu$ (after [24, Fig. 2]).

From Definitions 1 and 2, we infer that the operator $B A$ is

$$
B A f(u)=\int_{-\Delta u / 2}^{\Delta u / 2} f\left(u^{\prime}\right) \frac{\sin \left(\pi \Delta \mu\left(u-u^{\prime}\right)\right)}{\pi\left(u-u^{\prime}\right)} d u^{\prime} .
$$

The eigenfunctions of operator $B A$ are called prolate spheroidal functions. The following theorem [23], [26] states some properties of these functions and their eigenvalues.

Theorem 1: The operator $B A$ has countably many eigenvalues $\gamma_{n}, n \in \mathbb{N}$ such that

$$
\begin{aligned}
& 1 \geq \gamma \triangleq \gamma_{1} \geq \gamma_{2} \geq \gamma_{3} \geq \ldots \gamma_{n} \rightarrow 0 \\
& \sum_{n=1}^{\infty} \gamma_{n}=\Delta u \Delta \mu, \quad \sum_{n=1}^{\infty} \gamma_{n}^{2} \leq \Delta u \Delta \mu .
\end{aligned}
$$

The largest eigenvalue $\gamma$

1) is a concave and increasing function of the product $\Delta u \Delta \mu$

2) satisfies $\left.\gamma\right|_{\Delta u \Delta \mu=0}=0$ and $\lim _{\Delta u \Delta \mu \rightarrow \infty} \gamma=1$.

Furthermore, the eigenfunctions $e_{n}$ (prolate spheroidal functions) associated with the eigenvalues $\gamma_{n}$ have the following properties.

1) $\left\{e_{n} \mid n \geq 1\right\}$ is an orthonormal basis for the class of functions bandlimited to $[\Delta \mu / 2, \Delta \mu / 2]$.

2) $\left\{\gamma_{n}^{-1 / 2} A e_{n} \mid n \geq 1\right\}$ is an orthonormal basis for the class of functions space-limited to $[-\Delta u / 2, \Delta u / 2]$.

3) The functions $e_{n}$, suitably truncated and scaled, equal their Fourier transforms.

There is no analytical expression for $\gamma$ as a function of $\Delta u \Delta \mu$, but there are some upper bounds, such as $\gamma \leq \sqrt{\Delta u \Delta \mu}$. (See [1, eq. (3.5)].) A plot of $\gamma(\Delta u \Delta \mu)$ is provided in Fig. 1. Note that $\gamma$ approaches unity as the $\Delta u \Delta \mu$ product increases beyond a certain value. Dual results for the operator $\mathrm{AB}$ can also be stated but would not add anything for the purpose of this letter.

Having reviewed prolate spheroidal functions and the largest eigenvalue $\gamma$ of $B A$, we state the following key theorem from [24]:

Theorem 2: Let $\alpha \in[0,1]$ and $\beta \in[0,1]$ be defined by the equations

$$
\alpha^{2}=\frac{\int_{-\Delta u / 2}^{\Delta u / 2}|f(u)|^{2} d u}{\int_{-\infty}^{\infty}|f(u)|^{2} d u} \beta^{2}=\frac{\int_{-\Delta \mu / 2}^{\Delta \mu / 2}|F(\mu)|^{2} d \mu}{\int_{-\infty}^{\infty}|F(\mu)|^{2} d \mu} .
$$

The set of pairs $(\alpha, \beta)$ achievable for some function $f$ satisfying $\int_{-\infty}^{\infty}|f(u)|^{2} d u<\infty$ is the region defined by the inequality

$$
\cos ^{-1} \alpha+\cos ^{-1} \beta \geq \cos ^{-1} \sqrt{\gamma}
$$


together with $0 \leq \alpha, \beta \leq 1$ but excluding the points $(0,1)$ and $(1,0)$. For $\alpha \leq \sqrt{\gamma}$, the function achieving the boundary of this region is bandlimited to $[-\Delta \mu / 2, \Delta \mu / 2]$ and is equal to a linear combination of the family $\left\{e_{n} \mid n \geq 1\right\}$. For $\alpha \geq \sqrt{\gamma}$, the function achieving (5) with equality is given by the formula

$$
f(u)=\frac{\alpha}{\sqrt{\gamma}} A e_{1}(u)+\sqrt{\frac{1-\alpha^{2}}{1-\gamma}}\left(e_{1}(u)-A e_{1}(u)\right)
$$

where $e_{1}$ is the prolate spheroidal function having the largest eigenvalue $\gamma$.

Note that $\alpha^{2}$ and $\beta^{2}$ are the normalized energies contained in the intervals $[-\Delta u / 2, \Delta u / 2]$ and $[\Delta \mu / 2, \Delta \mu / 2]$, respectively. Therefore, $1-\alpha^{2}$ and $1-\beta^{2}$ are the normalized energies contained in the tails. Thus, the constraint (5) on how large $\alpha$ and $\beta$ can simultaneously be is also a constraint on how small the normalized energies contained in the tails in the two domains can simultaneously be.

In the following section, we use Theorem 2 to prove (4) and show that $\kappa=1-\sqrt{\gamma}$, therefore achieving our aim.

\section{PRoOF OF (4)}

We start with the observation that the left-hand side of (4) can be rewritten as

$$
\begin{aligned}
& \int_{|u|>\Delta u / 2}|f(u)|^{2} d u+\int_{|\mu|>\Delta \mu / 2}|F(\mu)|^{2} d \mu \\
& =\left(1-\alpha^{2}\right) \int_{-\infty}^{\infty}|f(u)|^{2} d u+\left(1-\beta^{2}\right) \int_{-\infty}^{\infty}|F(\mu)|^{2} d \mu \\
& =\left(2-\alpha^{2}-\beta^{2}\right) \int_{-\infty}^{\infty}|f(u)|^{2} d u
\end{aligned}
$$

thanks to Parseval's theorem. Assuming the energy of the signal $\int_{-\infty}^{\infty}|f(u)|^{2} d u$ is given, this implies we need to minimize $2-$ $\alpha^{2}-\beta^{2}$ over the achievable region of $(\alpha, \beta)$ pairs described in Theorem 2, in order to minimize the energy "left out" in the tails of the functions and the reconstruction error. More precisely, the problem we are trying to solve here can be formulated as

$$
\begin{aligned}
& \operatorname{argmax}_{(\alpha, \beta)} 2-\alpha^{2}-\beta^{2} \\
& \text { subject to } \cos ^{-1} \alpha+\cos ^{-1} \beta \geq \cos ^{-1} \sqrt{\gamma} .
\end{aligned}
$$

It is interesting that although the work of Landau, Pollak, and Slepian elegantly characterizes the tradeoff between $\alpha$ and $\beta$, they have no reason to, and therefore do not look at how large $\alpha^{2}+\beta^{2}$ can be under this constraint. Since we now see that the normalized reconstruction error is equal to $2-\left(\alpha^{2}+\beta^{2}\right)$, we become interested in how large $\alpha^{2}+\beta^{2}$ can be in order to obtain a lower bound for the error.

Let the result of minimization of $2-\left(\alpha^{2}+\beta^{2}\right)$ be denoted as $e_{\min }$. We see from (5) that any $\beta$ between 0 and 1 is achievable if $\alpha \leq \sqrt{\gamma}$. (This is because the function $\cos ^{-1}$ is decreasing and nonnegative.) For this part of the region of achievable pairs, we have

$$
2-\alpha^{2}-\beta^{2} \geq 2-\gamma-\beta^{2} \geq 2-\gamma-1=1-\gamma
$$

where the inequalities are achieved with equality for the pair $(\alpha, \beta)=(\sqrt{\gamma}, 1)$. To handle the case $\alpha>\sqrt{\gamma}$, we write

$$
\cos ^{-1} \beta \geq \cos ^{-1} \sqrt{\gamma}-\cos ^{-1} \alpha>0
$$

which can be expressed as

$$
\begin{aligned}
\beta & \leq \cos \left(\cos ^{-1} \sqrt{\gamma}-\cos ^{-1} \alpha\right) \\
& =\alpha \sqrt{\gamma}+\sin \left(\cos ^{-1} \alpha\right) \sin \left(\cos ^{-1} \sqrt{\gamma}\right) \\
& =\alpha \sqrt{\gamma}+\sqrt{1-\alpha^{2}} \sqrt{1-\gamma}
\end{aligned}
$$

since the cosine function is decreasing on the interval $[0, \pi / 2]$. Taking the square of both sides in (9), we obtain

$$
\beta^{2} \leq \alpha^{2}(2 \gamma-1)+2 \alpha \sqrt{1-\alpha^{2}} \sqrt{\gamma-\gamma^{2}}+1-\gamma
$$

from which

$$
2-\alpha^{2}-\beta^{2} \geq(1-\gamma)+2 \gamma\left(1-\alpha^{2}\right)-2 \alpha \sqrt{1-\alpha^{2}} \sqrt{\gamma-\gamma^{2}}
$$

follows. Combining (8) with (10), we get

$$
\begin{aligned}
& e_{\min }=\min \left\{\operatorname { m i n } _ { \alpha > \sqrt { \gamma } } \left\{(1-\gamma)+2 \gamma\left(1-\alpha^{2}\right)\right.\right. \\
& \left.\left.-2 \alpha \sqrt{1-\alpha^{2}} \sqrt{\gamma-\gamma^{2}}\right\}, 1-\gamma\right\} \\
& =\min _{\alpha>\sqrt{\gamma}}\left\{(1-\gamma)+2 \gamma\left(1-\alpha^{2}\right)-2 \alpha \sqrt{1-\alpha^{2}} \sqrt{\gamma-\gamma^{2}}\right\}
\end{aligned}
$$

where the last equation follows from $(1-\gamma)+2 \gamma\left(1-\alpha^{2}\right)-$ $\left.2 \alpha \sqrt{1-\alpha^{2}} \sqrt{\gamma-\gamma^{2}}\right|_{\alpha=1}=1-\gamma$. Since $\alpha \sqrt{1-\alpha^{2}}$ is increasing when $\alpha \in[0,1 / \sqrt{2}]$, the RHS of (10) is decreasing in the same interval. To observe how the RHS of (10) behaves when $\alpha \geq 1 / \sqrt{2}$, we compute the derivative

$$
\begin{aligned}
& \frac{\partial}{\partial \alpha}\left[(1-\gamma)+2 \gamma\left(1-\alpha^{2}\right)-2 \alpha \sqrt{1-\alpha^{2}} \sqrt{\gamma-\gamma^{2}}\right] \\
& \quad=-2\left(2 \alpha \gamma+\sqrt{\gamma-\gamma^{2}} \frac{1-2 \alpha^{2}}{\sqrt{1-\alpha^{2}}}\right)
\end{aligned}
$$

which is negative when

$$
\begin{gathered}
2 \alpha \gamma \geq \sqrt{\gamma-\gamma^{2}} \frac{2 \alpha^{2}-1}{\sqrt{1-\alpha^{2}}} \\
4 \alpha^{2} \gamma^{2} \geq\left(\gamma-\gamma^{2}\right) \frac{4 \alpha^{4}-4 \alpha^{2}+1}{1-\alpha^{2}} .
\end{gathered}
$$

Note that (13) is fully equivalent to (12) for $\alpha \geq 1 / \sqrt{2}$. Arranging the terms, (13) simplifies to

$$
\begin{array}{r}
4 \gamma \alpha^{4}-4 \gamma \alpha^{2}+\gamma-\gamma^{2} \leq 0 \\
4 \gamma\left(\alpha^{2}-\frac{1-\sqrt{\gamma}}{2}\right)\left(\alpha^{2}-\frac{1+\sqrt{\gamma}}{2}\right) \leq 0 .
\end{array}
$$

We conclude from (14) that the RHS of (10) is decreasing when $1 / 2 \leq \alpha^{2} \leq(1+\sqrt{\gamma}) / 2$ as well as the case when $\alpha^{2} \leq 1 / 2$. We also see from (14) that the RHS of (10) is no longer a decreasing function of $\alpha$ after $\alpha^{2}$ exceeds the threshold 
$(1+\sqrt{\gamma}) / 2$. Noting that

$$
\alpha_{\mathrm{opt}}=\sqrt{\frac{1+\sqrt{\gamma}}{2}} \geq \sqrt{\frac{\gamma+\gamma}{2}}=\sqrt{\gamma}
$$

we compute $e_{\min }$ as

$$
\begin{aligned}
e_{\min }= & {\left[(1-\gamma)+2 \gamma\left(1-\alpha^{2}\right)\right.} \\
& \left.-2 \alpha \sqrt{1-\alpha^{2}} \sqrt{\gamma-\gamma^{2}}\right]\left.\right|_{\alpha^{2}=(1+\sqrt{\gamma}) / 2} \\
= & 1-\gamma+2 \gamma \frac{1-\sqrt{\gamma}}{2}-2 \sqrt{\frac{1+\sqrt{\gamma}}{2}} \sqrt{\frac{1-\sqrt{\gamma}}{2}} \sqrt{\gamma-\gamma^{2}} \\
= & 1-\gamma \sqrt{\gamma}-\sqrt{\gamma}(1-\gamma) \\
= & 1-\sqrt{\gamma}
\end{aligned}
$$

which is achieved only when $\alpha_{\mathrm{opt}}^{2}=(1+\sqrt{\gamma}) / 2$ and

$$
\beta_{\mathrm{opt}}^{2}=2-\frac{1+\sqrt{\gamma}}{2}-e_{\mathrm{min}}=\frac{1+\sqrt{\gamma}}{2}=\alpha_{\mathrm{opt}}^{2} .
$$

Moreover, we see from Theorem 2 that $e_{\min }=1-\sqrt{\gamma}$ is achieved by the function

$$
\begin{aligned}
& f_{\text {opt }}(u)=\left[\frac{\alpha}{\sqrt{\gamma}} A e_{1}(u)+\sqrt{\frac{1-\alpha^{2}}{1-\gamma}}\right. \\
& \left.\cdot\left(e_{1}(u)-A e_{1}(u)\right)\right]\left.\right|_{\alpha^{2}=(1+\sqrt{\gamma}) / 2} \\
& =\sqrt{\frac{1+\sqrt{\gamma}}{2 \gamma}}\left[A e_{1}(u)+\frac{\sqrt{\gamma}}{1+\sqrt{\gamma}}\left(e_{1}(u)-A e_{1}(u)\right)\right] .
\end{aligned}
$$

This completes the proof of (4) with $\kappa=1-\sqrt{\gamma}$. This is a remarkably simple result showing how $\kappa$ approaches zero as $\gamma$ approaches unity.

Remark IV.1: A similar result obtained without using the setup of [24] appears in [1, eq. (3.4)]. In terms of our notation, that equation can be written as

$$
\sqrt{1-\alpha^{2}}+\sqrt{1-\beta^{2}} \geq 1-\gamma .
$$

But, the minimum value that the quantity $\sqrt{1-\alpha^{2}}+\sqrt{1-\beta^{2}}$ attains is $\sqrt{1-\gamma}>1-\gamma$. (The minimizers are the corner points $(\alpha, \beta)=(\sqrt{\gamma}, 1)$ and $(1, \sqrt{\gamma})$.) Hence, the bound (19) is not a tight one.

\section{DISCUSSION AND CONCLUSION}

Since we have a function that achieves the lower bound, this also means that the bound we have found is the tightest such bound possible. This was achieved by optimizing over the possible $(\alpha, \beta)$ pairs allowed by Theorem 2 .

The dependence of $\gamma$ on the product $\Delta u \Delta \mu$ was already given in Fig. 1. Therefore, the function $\kappa(\Delta u \Delta \mu)$ can be readily obtained and is plotted as the "theoretical limit" in Fig. 2. Note that this is a universal curve and does not depend on anything. It tells us that if we use a certain finite number of samples for representing a function, the normalized reconstruction error cannot be below the value $\kappa(\Delta u \Delta \mu)$ given by this curve.

Referring to Fig. 1, observe that we can roughly approximate $\gamma \approx \min (\Delta u \Delta \mu, 1)$, leading to $\kappa=1-\sqrt{\min (\Delta u \Delta \mu, 1)} \approx$

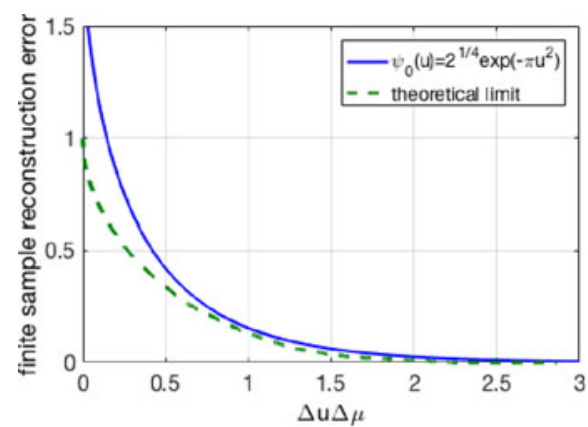

Fig. 2. Theoretical lower bound $\kappa=1-\sqrt{\gamma}$ for the truncation error and by virtue of (2) the reconstruction error, together with the actual reconstruction error for $f(u)=2^{1 / 4} e^{-\pi u^{2}}$.

$1-\min (\sqrt{\Delta u \Delta \mu}, 1)$. This means that for lower values of $\Delta u \Delta \mu$ the curve exhibits the dependence $\kappa \approx 1-\sqrt{\Delta u \Delta \mu}$, which is consistent with Fig. 2.

For comparison, we plotted in Fig. 2 the Pareto-optimal reconstruction error against the number of samples, for the zeroth-order Hermite-Gaussian function $\psi_{0}(u)=2^{1 / 4} e^{-\pi u^{2}}$ [12], alongside the theoretical limit $\kappa=1-\sqrt{\gamma}$. From the perspective of the classical Uncertainty Principle [24], [26], [31], [32], $\psi_{0}(u)$ is the function that is most simultaneously concentrated in both the space and frequency domains. It satisfies the Uncertainty Principle inequality with equality, meaning that the product of the standard deviations of the signal in the two domains is the smallest possible over all functions. In contrast, in this letter, the measure of being most simultaneously concentrated in both domains is how small the sum of the spatial and frequency truncation errors are [RHS of (2)]. We showed that the most concentrated function according to this measure is given by (18). Despite the different approaches, Fig. 2 shows that the difference between the theoretical limit achieved by (18) and the curve corresponding to $\psi_{0}(u)$ are not very different, and the difference becomes smaller as $\Delta u \Delta \mu>1$ increases.

It is worth contrasting the result of this letter with that of [12], where we also considered sampling of finite-energy signals with a finite number of samples. However, there we assumed that statistical knowledge of the ensemble of possible signals in the form of covariance functions was available. We showed that the expected value of the reconstruction error was approximately equal to the expected value of the energies left out (neglected) in the tails in the space and frequency domains. We further showed how to minimize this error by optimally choosing the space and frequency extents for a specified number of samples. All the results depend on the specified covariance functions. In this letter, no assumptions, statistical or otherwise, are made regarding the signals, other than that they have finite energy. We derive a universal curve, valid for all finite-energy functions, that provides a tight lower bound for the reconstruction error as a function of the number of samples used. In [12], the final curves are optimized over the space and frequency extents $\Delta u$ and $\Delta \mu$. Here, the final universal curve is optimized over $\alpha$ and $\beta$.

\section{ACKNOWLEDGMENT}

The work of H. M. Ozaktas was supported in part by the Turkish Academy of Sciences. 


\section{REFERENCES}

[1] D. L. Donoho and P. B. Stark, "Uncertainty principles and signal recovery," SIAM J. Appl. Math., vol. 49, no. 3, pp. 906-931, 1989.

[2] A. J. Jerri, "The Shannon sampling theorem-Its various extensions and applications: A tutorial review," Proc. IEEE, vol. 65, no. 11, pp. 15651596, Nov. 1977.

[3] P. L. Butzer and R. L. Stens, "Sampling theory for not necessarily bandlimited functions: A historical overview," SIAM Rev., vol. 34, no. 1, pp. 40 53, 1992.

[4] M. Unser, "Sampling-50 years after Shannon," Proc. IEEE, vol. 88, no. 4, pp. $569-587$, Apr. 2000

[5] P. P. Vaidyanathan, "Generalizations of the sampling theorem: Seven decades after Nyquist," IEEE Trans. Circuits Syst. I, Fundam. Theory Appl., vol. 48, no. 9, pp. 1094-1109, Sep. 2001.

[6] P. J. S. G. Ferreira, "On the approximation of nonbandlimited signals by nonuniform sampling series," in Proc. EUSIPCO, Eur. Signal Proc. Conf., 1996, pp. $1567-1570$

[7] J. L. Brown, "Estimation of energy aliasing error for nonbandlimited signals," Multdimensional Syst. Signal Process., vol. 15, no. 1, pp. 51-56, 2001.

[8] J. L. Brown, "On the error in reconstructing a non-bandlimited function by means of the bandpass sampling theory," J. Math. Anal. Appl. vol. 18, no. 1 , pp. $75-84,1967$.

[9] A. Özçelikkale and H. M. Ozaktas, "Representation of optical fields using finite numbers of bits," Opt. Lett., vol. 37, pp. 2193-2195, 2012.

[10] A. Özçelikkale and H. M. Ozaktas, "Beyond Nyquist Sampling: A costbased approach," J. Opt. Soc. Amer. A, vol. 30, no. 4, pp. 645-655, 2013.

[11] A. Özçelikkale and H. Ozaktas, "Optimal representation of non-stationary random fields with finite numbers of samples: A linear MMSE framework," Digit. Signal Process., vol. 23, no. 5, pp. 1602-1609, 2013.

[12] T. C. Gulcu and H. M. Ozaktas, "Choice of sampling interval and extent for finite-energy signals," IEEE Trans. Signal Process., vol. 65, no. 7, pp. 1751-1761, Apr. 2017.

[13] F. Gori and G. Guattari, "Shannon number and degrees of freedom of an image," Opt. Commun., vol. 7, no. 2, pp. 163-165, 1973.

[14] D. Mendlovic and A. W. Lohmann, "Space bandwidth product adaptation and its application to superresolution: Fundamentals," J. Opt. Soc. Amer A, vol. 14, no. 3, pp. 558-562, 1997.

[15] H. M. Ozaktas and F. S. Oktem, "Phase-space window and degrees of freedom of optical systems with multiple apertures," J. Opt. Soc. Amer. A, vol. 30, no. 4, pp. 682-690, 2013.

[16] A. Poon, R. Brodersen, and D. Tse, "Degrees of freedom in multipleantenna channels: A signal space approach," IEEE Trans. Inf. Theory, vol. 51, no. 2, pp. 523-536, Feb. 2005.
[17] C. Wang, T. Gou, and S. A. Jafar, "Subspace alignment chains and the degrees of freedom of the three-user MIMO interference channel," IEEE Trans. Inf. Theory, vol. 60, no. 5, pp. 2432-2479, May 2014.

[18] J. Xie and S. Ulukus, "Secure degrees of freedom of one-hop wireless networks," IEEE Trans. Inf. Theory, vol. 60, no. 6, pp. 3359-3378, Jun. 2014.

[19] F. S. Oktem and H. M. Ozaktas, "Exact relation between continuous and discrete linear canonical transforms," IEEE Signal Process. Lett., vol. 16, no. 8, pp. 727-730, Aug. 2009.

[20] F. S. Oktem and H. M. Ozaktas, "Equivalence of linear canonical transform domains to fractional Fourier domains and the bicanonical width product: A generalization of the space-bandwidth product," J. Opt. Soc. Amer. A, vol. 27, no. 8, pp. 1885-1895, 2010.

[21] H. M. Ozaktas, A. Koc, I. Sari, and M. A. Kutay, "Efficient computation of quadratic-phase integrals in optics," Opt. Lett., vol. 31, no. 1, pp. 35-37, 2006.

[22] A. Koc, H. M. Ozaktas, C. Candan, and M. A. Kutay, "Digital computation of linear canonical transforms," IEEE Trans. Signal Process., vol. 56, no. 6, pp. 2383-2394, Jun. 2008.

[23] D. Slepian and H. O. Pollak, "Prolate spheroidal wave functions, Fourier analysis and uncertainty-I," Bell Syst. Tech. J., vol. 40, no. 1, pp. 43-64, 1961

[24] H. J. Landau and H. O. Pollak, "Prolate spheroidal wave functions, Fourier analysis and uncertainty-II," Bell Syst. Tech. J., vol. 40, no. 1, pp. 65-84, 1961.

[25] H. J. Landau and H. O. Pollak, "Prolate spheroidal wave functions, Fourier analysis and uncertainty-III: The dimension of the space of essentially time and bandlimited signals," Bell Syst. Tech. J., vol. 41, no. 4, pp. 12951336, 1962.

[26] H. Dym and H. P. McKean, Fourier Series and Integrals. New York, NY, USA: Academic, 1972.

[27] I. C. Moore and M. Cada, "Prolate spheroidal wave functions, an introduction to the Slepian series and its properties," Appl. Comput. Harmon. Anal., vol. 16, no. 3, pp. 208-230, 2004.

[28] E. Pauwels and M. de Gosson, "On the prolate spheroidal wave functions and Hardys uncertainty principle," J. Fourier Anal. Appl., vol. 20, no. 3, pp. 566-576, 2014

[29] A. Karoui and A. Souabni, "Weighted finite Fourier transform operator: Uniform approximations of the eigenfunctions, eigenvalues decay and behaviour," J. Scientific Comput., vol. 71, no. 2, pp. 547-570, 2017.

[30] A. Bonami and A. Karoui, "Approximations in Sobolev spaces by prolate spheroidal wave functions," Appl. Comput. Harmon. Anal., vol. 42, pp. 361-377, 2017

[31] A. Papoulis, Signal Analysis. New York, NY, USA: McGraw-Hill, 1977.

[32] H. M. Ozaktas, Z. Zalevsky, and M. A. Kutay, The Fractional Fourier Transform With Applications in Optics and Signal Processing. Hoboken, NJ, USA: Wiley, 2001 